\title{
Variability in adherence to clinical practice guidelines and recommendations in COPD outpatients: a multi-level, cross-sectional analysis of the EPOCONSUL study
}

Myriam Calle Rubio ${ }^{1 *}$ (D) José Luis López-Campos ${ }^{2,3}$, Juan J. Soler-Cataluña ${ }^{4}$, Bernardino Alcázar Navarrete ${ }^{5}$, Joan B. Soriano ${ }^{6}$, José Miguel Rodríguez González-Moro ${ }^{7}$, Manuel E. Fuentes Ferrer ${ }^{8}$, Juan Luis Rodríguez Hermosa ${ }^{1}$ and On behalf of the EPOCONSUL Study

\begin{abstract}
Background: Clinical audits have reported considerable variability in COPD medical care and frequent inconsistencies with recommendations. The objectives of this study were to identify factors associated with a better adherence to clinical practice guidelines and to explore determinants of this variability at the the hospital level.

Methods: EPOCONSUL is a Spanish nationwide clinical audit that evaluates the outpatient management of COPD. Multilevel logistic regression with two levels was performed to assess the relationships between individual and disease-related factors, as well as hospital characteristics.

Results: A total of 4508 clinical records of COPD patients from 59 Spanish hospitals were evaluated. High variability was observed among hospitals in terms of medical care. Some of the patient's characteristics (airflow obstruction, degree of dyspnea, exacerbation risk, presence of comorbidities), the hospital factors (size and respiratory nurses available) and treatment at a specialized COPD outpatient clinic were identified as factors associated with a better adherence to recommendations, although this only explains a small proportion of the total variance.

Conclusion: To be treated at a specialized COPD outpatient clinic and some intrinsic patient characteristics were factors associated with a better adherence to guideline recommendations, although these variables were only explaining part of the high variability observed among hospitals in terms of COPD medical care.
\end{abstract}

Keywords: Chronic obstructive pulmonary disease, Clinical audit, Medical care, Clinical practice guidelines, Adherence to recommendations

\section{Background}

Chronic obstructive pulmonary disease (COPD) is one of the most frequent reasons for seeking medical attention and accounts for $10 \%$ of primary care and $30 \%$ of outpatient respiratory care visits [1]. Patients with this condition have a high morbidity and mortality $[2,3]$. For these reasons, there are a number of clinical practice guidelines $(\mathrm{CPG})$ aimed to systemize

\footnotetext{
* Correspondence: mcallerubio@gmail.com

${ }^{1}$ Pulmonary Department, Research Institute of Hospital Clínico San Carlos (IdISSC), Faculty of Medicine, University Complutense of Madrid, C/ Martin Lagos s/n, 28040 Madrid, Spain

Full list of author information is available at the end of the article
}

medical care for COPD [4-7]. However, the real-life implementation of these CPG is low $[8,9]$.

Clinical audits have emerged as an overarching tool to measure the adequacy of clinical practice and feedback is being used to improve health care [10]. For more than 12 years, some countries have been auditing the quality of their inhospital COPD management in a systematic way [11-13]. However, we have less evidence regarding outpatient care, and the few existing studies only explored certain aspects, such as the diagnosis or the prescription pattern, showing us outpatient care is far from perfect [14-18] with considerable variability in COPD medical care and frequent inconsistencies with CPG recommendations. 
The EPOCONSUL study is the first national audit to evaluate the adequacy of medical care according to CPG in Spain in COPD patients treated at outpatient respiratory clinics. The study confirmed significant variations in adherence to CPG recommendations between centers [19]. The objective of our work has been analyze the variability and to identify factors associated with adherence to current recommendations for COPD clinical practice guidelines for outpatients in Spain.

\section{Methods}

The methodology of the EPOCONSUL audit has been extensively described elsewhere [19]. Briefly, the COPD audit promoted by the Spanish Society of Pneumology and Thoracic Surgery (SEPAR) was designed to evaluate clinical practice as well as clinical and organizational factors related to managing patients with COPD across Spain. It was designed as an observational non-interventional cross-sectional study. Recruitment was intermittent during the year (May 2014-May 2015). Every 2 months each investigator recruited clinical records of the first 10 patients identified as diagnosed with COPD and seen in the outpatient respiratory clinic. Subsequently, patients identified were reevaluated to determine if they met the inclusion/exclusion criteria described in Appendix 1 . The sampling process was detailed in an epidemiology flow chart and described in Appendix 2.

The information collected was historical in nature for the clinical data of the last and previous visits, and the information about hospital resources was concurrent.

As described in the methodological research paper [19], in order to evaluate the degree of current CPG implementation of the main statements according to the 2012 Spanish National Guidelines for COPD care (GesEPOC) and the 2013 Global initiative for chronic Obstructive Lung Disease (GOLD), we established fulfilling $\geq 50 \%$ of criteria for good clinical practice evaluated in each category (clinical evaluation of the patient, COPD evaluation and therapeutic intervention) as the cut-off point.

From the 175 public hospitals in the National Health System invited from the Spanish Society of Pneumology and Thoracic Surgery, 59 participated (33.3\%). The estimated reference population for the EPOCONSUL study was 18,104,350 inhabitants, representing $39 \%$ of the Spanish population. The distribution of hospitals in the different regions and the population covered by those hospitals are detailed in Appendix 3 and participating investigators are included in Appendix 4.
In order to compare hospitals, these were divided in two types of center according to their size (small or large) as measured by: the number of beds per center $\geq 500$, the number of inpatient respiratory beds $\geq 20$, the number of pulmonology staff members $\geq 5$, and the number of annual outpatient respiratory visits $\geq 10,000$. All the criteria are necessary to be considered large.

The protocol was approved by the Ethics Committee of the Hospital Clínico San Carlos (Madrid, Spain; internal code 14/030-E). Additionally, according to current research laws in Spain, the ethics committee at each participating hospital evaluated and agreed to the study protocol. The need for informed consent was waived because ours is a clinical audit, beside the non-interventional nature of the study, the anonymization of data and the need to blindly evaluate the clinical performance. This circumstance was clearly explained in the protocol, and the ethical committees approved this procedure. To avoid modifications to the usual clinical practice and preserve the blinding of the clinical performance evaluation, the medical staff responsible for the outpatient respiratory clinic was not informed about the audit. Data was entered remotely at each participating location to a centrallycontrolled server.

\section{Statistical analysis}

Descriptive results are presented both at the patient and hospital level. Qualitative variables were summarized by their frequency distribution as well as quantitative variables by their median, interquartile range (IQR) and minimum-maximum. The differences between hospital resources and characteristics according to size (small vs large) were evaluated using $\chi^{2}$ tests for categorical data, while the non-parametric MannWhitney test was used for continuous data. Significance of variability by area/hospital was explored by the Kruskal-Wallis or chi-square tests.

With regard to missing data, after performing data cleansing to identify and correct missing and extremely unlikely values, the data was included in the analysis as missing information.

Three dependent variables were generated to evaluate the degree of CPG implementation; criteria of good clinical practice were categorized into: fulfilling three criteria at the clinical evaluation, fulfilling four criteria at the COPD evaluation, and fulfilling three criteria at the therapeutic intervention.

The association between each independent variable (patient characteristics, hospital resources and work organization) and each of the dependent variables was assessed by calculating the crude odds ratio (OR) via multilevel bivariate regression analysis. Each multilevel analysis included two levels: the individual or 
patient level (level 1), and the hospital level (level 2). It was developed in four consecutive steps: (1) Model 1 (empty model) which included only the dependent variable and the hospital-cluster effect; (2) random effects Model 2, which included the hospital variables; (3) random effects Model 3, which included the patient variables; (4) random effects Model 4, which included the patient and hospital variables in order to obtain an overall multivariable model. Candidate predictors with a value of $p<0.10$ in the univariate analysis were accepted for inclusion in the multivariate analysis in model 2 and 3. Variables were removed from the model when the $p$-value exceeded 0.10 and were kept in the final model when less than 0.05 . The independent predictor variables included in Model 4 were those selected in the last step in Models 2 and 3. The coefficients of the predictor variables were transformed into OR, with 95\% confidence intervals (CI).

The hospital cluster effect was evaluated and quantified by two indicators: 1) the intra-cluster correlation coefficient (ICC) which represents the proportion of the variance attributable to the clustering effect and 2) the median odds ratio (MOR). The MOR can be interpreted as the median increased odds of reaching the outcome if an individual was admitted to another hospital with a greater risk of that outcome.

All analyses were performed using STATA 12.0 software. Statistical significance was assumed as $p<0.05$.

\section{Results}

A total of 17,893 clinical records of patients treated in outpatient respiratory clinics were evaluated during the study period and 5726 clinical records of patients presumably diagnosed with COPD were selected. Of them, 4508 patients were audited from 59 hospitals, for having all the inclusion criteria and none of the exclusion criteria. The sampling process was detailed in an epidemiology flow chart and described in Appendix 2.

\section{Center characteristics}

The hospital characteristics and respiratory unit resources are summarized in Table 1. Large hospitals constituted $54 \%$ of centers. The majority participating centers were public (93.2\%), university hospitals (83.1\%) and had a pulmonary resident available (67.8\%). Although the larger hospitals had more staff, the length of the outpatient follow-up visit was similar. There were few centers with a specialized COPD outpatient clinic $(47.5 \%)$ and outpatient respiratory nursing clinic (45.8\%), regardless of hospital size.

\section{Audited patient characteristics and clinical conditions}

The main characteristics of the patients evaluated are presented in Table 2.

\section{Adequacy of medical care according to CPG}

Adherence to the main CPG statements is summarized in Table 3. There was a significant variation between hospitals, with a better adherence to the statements in the clinical evaluation category, with three out of six criteria fulfilled in $65.5 \%$ of the patients.

\section{Adherence to CPG recommendations based on patient and center characteristics}

The bivariate association between adherence to the main CPG statements and the variables related to hospital and patient characteristics is summarized in Appendix 5. A major number of the patient-level variables were associated with adherence, whereas the majority of centerlevel variables were not.

\section{Multilevel variability analysis of adherence to CPG recommendations}

For the adherence to the statements in the clinical evaluation category, fulfillment of at least three criteria, the percentage of the total variability attributable to the hospital-cluster effect was $36 \%$. The empty model exhibited a significant cluster effect $(\mathrm{ICC}=0.36)$ and cluster heterogeneity $(\mathrm{MOR}=3.73)$. In the adjusted model, being an active smoker, having a Charlson index $\geq 3$, undergoing $\geq 1$ hospitalization for COPD in the past year and being treated at a specialized COPD outpatient clinic was positively associated. Only one variable linked to the hospital level (large hospital) was retained in the model as a predictor, but was unfavorable (Table 4). The inclusion of all predictors further reduced the residual between-hospital cluster variability. The ICC and MOR dropped to 0.31 and 3.26, respectively (Table 4). Some unrecorded values (COPD phenotype missing) showed significant associations, which is naturally open to interpretation.

For COPD evaluation category, fulfillment of at least four criteria, the empty model displayed an ICC of 0.30 and a MOR of 3.13 (Table 4). In the adjusted model, an age of $\leq 55$ years, FEV $1<50 \%$, dyspnea $\geq 2$ MRC-m and being treated at a specialized COPD outpatient clinic were positively associated with better adherence to CPG recommendations. However, being male and having a Charlson index $\geq 3$ were retained as predictors of worse adherence. Some unrecorded values (COPD phenotype missing, dyspnea missing, or level of dyspnea not referred to) showed a significant negative association. Only one variable linked to the hospital level (i.e. respiratory ward availability) was retained as a predictor of better adherence. The inclusion of this predictor further reduced the between-hospital cluster variability. The ICC and MOR dropped to 0.24 and 2.67 , respectively. 
Table 1 Characteristics of the participating hospitals and resources of the respiratory units

\begin{tabular}{|c|c|c|c|c|}
\hline & All & Small hospital & Large hospital & $P^{+}$ \\
\hline Number of participating hospitals, $n$ & 59 & 27 & 32 & \\
\hline Public hospital (\%) & 93.2 & 85.2 & 100 & 0.039 \\
\hline University hospital (\%) & 83.1 & 63 & 100 & $<0.001$ \\
\hline Beds per center $\geq 500(\%)$ & 62.7 & 18.5 & 100 & $<0.001$ \\
\hline Beds per center, median (P25-75) & $651(349-943)$ & $332(231-436)$ & $903(702-1199)$ & $<0.001$ \\
\hline Hospital with a respiratory ward (\%) & 83.1 & 63 & 100 & $<0.001$ \\
\hline Number of inpatient respiratory beds $\geq 20(\%)$ & 83.7 & 52.9 & 100 & $<0.001$ \\
\hline Number of pulmonology staff members $\geq 5(\%)$ & 81.4 & 59.3 & 100 & $<0.001$ \\
\hline Number of pulmonology staff members, median (P25-75) & $10(5-13)$ & $5(2-8)$ & $13(10-16)$ & $<0.001$ \\
\hline Pulmonology residents available (\%) & 67.8 & 33.3 & 96.9 & $<0.001$ \\
\hline Number of annual outpatient respiratory visits, median (IQR) & $15,447(12004-25,680)$ & $12,004(4355-13,556)$ & $23,985(16070-27,838)$ & $<0.001$ \\
\hline Number of annual outpatient respiratory visits $\geq 10,000(\%)$ & 81.4 & 59.3 & 100 & $<0.001$ \\
\hline$\geq 15$ min of follow-up at general outpatient respiratory visit (\%) & 44.1 & 48.1 & 40.6 & 0.562 \\
\hline Specialized COPD outpatient clinic available (\%) & 47.5 & 40.7 & 53.1 & 0.343 \\
\hline$\geq 15$ min of follow-up at specialized COPD outpatient visit (\%) & 64.4 & 74.1 & 56.3 & 0.154 \\
\hline Outpatient respiratory nursing clinic availability (\%) & 45.8 & 44.4 & 46.9 & 0.852 \\
\hline \multicolumn{5}{|l|}{ Functional respiratory laboratory available (\%) } \\
\hline - Spirometry & 100 & 100 & 100 & 1 \\
\hline - Diffusing capacity & 100 & 100 & 100 & 1 \\
\hline - Plethysmography & 100 & 100 & 100 & 1 \\
\hline - Respiratory muscle strength & 84.7 & 66.7 & 100 & $<0.001$ \\
\hline - 6MWT available & 94.9 & 88.9 & 100 & 0.090 \\
\hline - Cardiopulmonary exercise testing available & 62.7 & 40.7 & 81.3 & 0.001 \\
\hline Inhalation technique educational program available (\%) & 30.5 & 15.6 & 48.1 & 0.007 \\
\hline Respiratory rehabilitation program available (\%) & 74.6 & 66.7 & 81.3 & 0.2 \\
\hline - Hospital-based & 61.4 & 61.1 & 61.5 & 0.617 \\
\hline - Home-based & 6.8 & 11.1 & 3.8 & \\
\hline - Mixed & 31.8 & 27.8 & 34.6 & \\
\hline
\end{tabular}

Data are presented as median (Cl 95\%), unless stated otherwise. Dichotomous variables are expressed as number and/or percent. $p^{\dagger}$ calculated by the Kruskal-Wallis or Chi-square test, depending on the nature of the variable

For therapeutic intervention category, fulfillment of at least three criteria, the empty model displayed an ICC of 0.52 and a MOR of 6.09. A Charlson index $\geq 3$, undergoing $\geq 1$ hospitalizations in the past year, being treated at a specialized COPD outpatient clinic, and outpatient respiratory nursing clinic availability were associated with better adherence to the recommendations. Meanwhile, being male, being $\leq 55$ years old and being a university hospital were all associated with worse adherence. The inclusion of these predictors further reduced the between-hospital cluster variability. The ICC and MOR dropped to 0.44 and 4.74 , respectively (Table 4).

\section{Discussion}

The present study constitutes one of the few research papers in the literature that analyze the variability in adherence to current recommendations for COPD clinical practice guidelines for outpatients in Spain. In our analysis, we aimed to study the variables associated with this variability.

This study shows that accounting for the hospital cluster effect, the patient-level and hospital-level predictor variables, partly reduced the unexplained between-hospital variation in adherence. Additionally, it identified a number of variables as predictors of better adherence at the patient and hospital levels. Most predictors were linked to patient characteristics (patient-level) and the type of respiratory clinic in which the patient was treated (general clinic or specialized COPD outpatient clinic).

Being treated at a specialized COPD outpatient clinic was associated with a higher likelihood of adherence to guidelines in the three categories evaluated, and was considered to be of greater importance, compared with the cluster effect, in explaining the between-hospital outcome variations. This is an 
Table 2 Clinical characteristics of the audited cases

\begin{tabular}{|c|c|c|c|c|c|}
\hline & Patient & & Hospitals & & $p^{+}$ \\
\hline & $\bar{N}$ & $\%$ or median (IQR) & Median & IQR & \\
\hline Sex (male), (\%) & 4.508 & 86 & 87.5 & $82.1-93.2$ & $<0.001$ \\
\hline Age (years), median (P25-75) & 4.508 & $69.7(63-77.7)$ & 70 & 69-72 & $<0.001$ \\
\hline$\leq 55(\%)$ & & 8.5 & 8.2 & $5.8-11.7$ & \\
\hline $56-69(\%)$ & & 38.7 & 38.1 & $30-42.6$ & \\
\hline$\geq 70(\%)$ & & 52.8 & 53.3 & $47.1-61.7$ & \\
\hline Pack-years, median (P25-75) & 4.508 & $47(34-70)$ & 45 & $40-51$ & $<0.001$ \\
\hline Active smokers, (\%) & 4.508 & 23.1 & 22 & $18-29$ & $<0.001$ \\
\hline BMl kg/m2, median (P25-75) & 4.508 & $28.0(24.4-31.1)$ & 27.8 & $26.6-28.5$ & 0.03 \\
\hline$\leq 21(\%)$ & & 7.1 & 6.7 & $4.1-9.2$ & \\
\hline $22-29(\%)$ & & 60.8 & 58.8 & $56.1-64$ & \\
\hline$\geq 30(\%)$ & & 32.1 & 31.4 & $26.2-37.7$ & \\
\hline Charlson index, median (P25-75) & 4.508 & $2(1-4)$ & 2 & $2-3$ & $<0.001$ \\
\hline$\geq 3(\%)$ & & 44.9 & 44.5 & $40-56.6$ & \\
\hline Dyspnea (MRC-m) & 4.508 & & & & $<0.001$ \\
\hline $0+1(\%)$ & & 27.3 & 23.8 & $11.6-44.5$ & \\
\hline$\geq 2(\%)$ & & 41.4 & 38.3 & $28.3-54$ & \\
\hline Missing (\%) & & 13.2 & 8.9 & $1.6-21.6$ & \\
\hline Level of dyspnea not referred to (\%) & & 18.1 & 11.6 & $3.3-30$ & \\
\hline CAT questionnaire >10, (\%) & 869 & 62.4 & 64 & $47.9-83.8$ & $<0.001$ \\
\hline Chronic bronchitis criteria, (\%) & 4.508 & 41.7 & 41 & $28.3-51$ & $<0.001$ \\
\hline Chronic colonization, (\%) & 4.508 & 6.0 & 5 & $3.2-8.3$ & $<0.001$ \\
\hline Symptoms suggestive of asthma,(\%) & 4.508 & 26.5 & 18.3 & $10.8-35$ & $<0.001$ \\
\hline \% FEV1, median (P25-75) & 4.508 & $50(37-63)$ & 51 & $47-54$ & 0.03 \\
\hline$<50 \%$ & & 49.1 & 45.5 & $41.5-53.3$ & \\
\hline $50-64 \%$ & & 28 & 28.5 & $22.3-31.7$ & \\
\hline$\geq 65 \%$ & & 22.9 & 23.8 & $15-30$ & \\
\hline Number of moderate/severe exacerbations in the last year, median (P25-75) & 3.196 & $1.1(0-2)$ & 1 & $0-1$ & 0.03 \\
\hline Number of hospital admissions in the last year, median (P25-75) & 4508 & $0.5(0-1)$ & 0 & $0-0$ & 0.03 \\
\hline BODE value, median (P25-75) & 632 & $3.9(3-5)$ & 4.5 & $3-5.5$ & $<0.001$ \\
\hline GOLD group & 985 & & & & $<0.001$ \\
\hline$A(\%)$ & & 22.7 & 14.3 & $0-25.9$ & \\
\hline B (\%) & & 18.7 & 16.7 & $0-24.1$ & \\
\hline$C(\%)$ & & 18.7 & 20 & $9.8-33.3$ & \\
\hline $\mathrm{D}(\%)$ & & 39.9 & 40 & 23.5-55.6 & \\
\hline GesEPOC Phenotype & 4.508 & & & & $<0.001$ \\
\hline - Non-exacerbator, (\%) & & 27.5 & 24.4 & $11.4-28$ & \\
\hline - Exacerbator, (\%) & & 18.8 & 15.7 & $3.4-22$ & \\
\hline - Missing, (\%) & & 53.7 & 52.3 & $44-58.9$ & \\
\hline LAMA monotherapy, (\%) & 4.391 & 10.0 & 10 & $4.8-15.3$ & 0.03 \\
\hline LAMA-LABA combination, (\%) & 4.391 & 22.7 & 20.3 & $14.5-27.9$ & $<0.001$ \\
\hline LABA+ ICS combination, (\%) & 4.391 & 7.7 & 6.7 & $3.4-9.8$ & 0.03 \\
\hline Triple therapy (LAMA + LABA + CSI), (\%) & 4.391 & 49.1 & 50.8 & $39.3-60.3$ & $<0.001$ \\
\hline Long-term oxygen therapy, (\%) & 4.508 & $26.6 \%$ & 25 & $17.1-33.3$ & 0.03 \\
\hline Home ventilation, (\%) & 4.508 & $7.5 \%$ & 5 & $2.5-11.6$ & $<0.001$ \\
\hline Respiratory rehabilitation, (\%) & 4.508 & 9 & 5 & $0-11.8$ & $<0.001$ \\
\hline Respiratory care follow-up (years), (\%) & 4.508 & $4(2-7)$ & 4 & $3.5-5$ & 0.03 \\
\hline
\end{tabular}

Dichotomous variables are expressed as $n$ and percentage. Average value expressed as median (P25-75). The variability was expressed using the interquartile range (IQR) of median. ${ }^{\dagger}$ Calculated for the variability between centers using test de Kruskal-Wallis or chi-square test, depending on the nature of the variable

Abbreviations: LABA long-acting beta-2 agonists, LAMA long-acting antimuscarinic agents, ICS inhaled corticosteroids, GOLD Global Initiative for Chronic Obstructive Lung Disease, GesEPOC Spanish National Guidelines for COPD, CAT COPD Assessment Test 
Table 3 Adherence to recommendations (GOLD and GesEPOC) evaluated in the study and classified in three categories: clinical evaluation of the patient, COPD evaluation and therapeutic interventions. The number of criteria or quality standards fulfilled was analyzed in each category

\begin{tabular}{|c|c|c|c|c|c|}
\hline $\begin{array}{l}\text { Criteria of good clinical practice } \\
\text { evaluated in EPOCONSUL }\end{array}$ & $\begin{array}{l}\mathrm{N} \text { of criteria } \\
\text { fulfilled }\end{array}$ & $\begin{array}{l}\text { Patients } \\
(N=4.508) \%\end{array}$ & $\begin{array}{l}\text { Hospitals } \\
(N=59) \text { Median }\end{array}$ & $\begin{array}{l}\text { Inter-hospital } \\
\text { range }\end{array}$ & $p^{\dagger}$ \\
\hline \multicolumn{6}{|l|}{ Clinical evaluation category } \\
\hline \multirow{2}{*}{$\begin{array}{l}\text { 1. Was degree of dyspnea evaluated during current visit? } \\
\text { 2. Was the number of hospital admissions in the last } 12 \text { months } \\
\text { collected during current visit? }\end{array}$} & 6 criteria & 18.3 & 14.6 & $0-100$ & $<0.001$ \\
\hline & $>3$ criteria & 65.5 & 70 & $11.7-100$ & $<0.001$ \\
\hline $\begin{array}{l}\text { 3. Was the number of moderate or severe exacerbations in the } \\
\text { last } 12 \text { months collected during current visit? } \\
\text { 4. Was current smoking habit information collected? } \\
\text { 5. Was regular exercise data collected during current visit? } \\
\text { 6. Are comorbidities identified in the clinical record? }\end{array}$ & $\leq 3$ criteria & 34.5 & 30 & $0-88.3$ & $<0.001$ \\
\hline \multicolumn{6}{|l|}{ COPD evaluation category } \\
\hline \multirow{3}{*}{$\begin{array}{l}\text { 1. Alfa-1-antitrypsin serum level determination available? } \\
\text { 2. COPD severity defined in the report? } \\
\text { 3. COPD GOLD type defined in the report? } \\
\text { 4. COPD phenotype according to GesEPOC defined in the report? } \\
\text { 5. GMWT carried out on any occasion? } \\
\text { 6. Diffusion capacity measured on any occasion? } \\
\text { 7. Lung volumes measured on any occasion? } \\
\text { 8. Chest CT scan carried out on any occasion in exacerbator } \\
\text { phenotype? }\end{array}$} & 8 criteria & 1.5 & 0 & $0-14.6$ & $<0.001$ \\
\hline & $>4$ criteria & 30.1 & 27 & $0-89.3$ & $<0.001$ \\
\hline & $\leq 4$ criteria & 69.9 & 73 & $10.7-100$ & $<0.001$ \\
\hline \multicolumn{6}{|l|}{ Therapeutic intervention category } \\
\hline \multirow{3}{*}{$\begin{array}{l}\text { 1. Is treatment adherence evaluated in any way? } \\
\text { 2. Is inhalation technique evaluated in any way? } \\
\text { 3. Is Pneumococcal vaccination recommended? } \\
\text { 4. Is exercise advised during the visit? } \\
\text { 5. Have arterial blood gases been measured on any occasion } \\
\text { in patients on long-term oxygen therapy? } \\
\text { 6. Is a specific intervention for smoking cessation for active smokers offered? }\end{array}$} & 6 criteria & 9.3 & 3.3 & $0-45.1$ & $<0.001$ \\
\hline & $>3$ criteria & 22.4 & 12.5 & $0-100$ & $<0.001$ \\
\hline & $\leq 3$ criteria & 77.6 & 87.5 & $0-100$ & $<0.001$ \\
\hline
\end{tabular}

Dichotomous variables are expressed as $n$ and percentage. The variability between centers was expressed using the inter-hospital range (min-max). $p^{\dagger}$ was calculated for the variability between centers using the Kruskal-Wallis or Chi-square tests, depending on the nature of the variable

interesting result, since less than half of the centers had specialized COPD outpatient clinics. In addition, the time available at specialized COPD outpatient clinics to treat the patient was the same as the general outpatient respiratory visit and there was no support nurse. Consequently, this could be considered a proxy for the experience, knowledge and interest of department physicians in the management of COPD patients.

Also, some unrecorded values (COPD phenotype missing and level of dyspnea missing) showed a statistically significant negative association, which are naturally open to interpretation.

The clinical COPD phenotype according to the Spanish National Guideline for COPD (GesEPOC) was collected in $46.3 \%$ of the audited patients.

Only 2 (outpatient respiratory nursing clinic and a respiratory ward availability) of the 46 hospital-level variables examined were retained in the model associated with a higher likelihood of implementing CPG recommendations. On the contrary, being a university hospital or large hospital were negatively associated factors. Nevertheless, given the small amount of cluster variability left unexplained in the analysis, it is unlikely that relevant hospital-level variables were not revealed. It's possible that this finding is the result of a relative small hospital sample size $(N=59)$. Thus, medical care in COPD does not require complex interventions and the majority of respiratory units offered a functional respiratory laboratory. We must consider the fact that this study did not include information about work organization such as COPD clinical management protocol availability or electronic/ digital information availability. It also did not include the number of respiratory physicians or respiratory nurses available in the area around the clinic or the professional experience of treating physicians, which might explain a proportion of the total variance due to the center effect.

Our findings are similar to those of previous studies that have demonstrated significant variability in the processes of COPD care. In the European COPD Audit [13], a considerable variability in recommendation guideline suitability was described and only hospital characteristics were related to a minority of indicators. The adherence to guidelines also varied with hospital size, but the differences were small and inconsistent. Previous studies have shown adherence to clinical guidelines was a strong predictor of a favorable outcome. Roberts et al. [11] have suggested that a 
Table 4 Multilevel logistic regression models of the variability in adherence to good clinical practice criteria for three categories: clinical evaluation of the patient, disease evaluation and therapeutic interventions

\begin{tabular}{|c|c|c|c|c|c|}
\hline & $\begin{array}{l}\text { Intra-class } \\
\text { correlation (ICC) }\end{array}$ & $\begin{array}{l}\text { Median Odds } \\
\text { Ratio (MOR) }\end{array}$ & Variables & $\begin{array}{l}\text { Adjusted OR } \\
(95 \% \mathrm{Cl})\end{array}$ & p \\
\hline \multicolumn{6}{|c|}{ Adherence to good clinical practice criteria in clinical evaluation ( $\geq 3$ criteria fulfilled) } \\
\hline Empty model 1 & 0.36670 & 3.73040 & & & \\
\hline Model 2: center variables ${ }^{1}$ & 0.31866 & 3.26487 & & & \\
\hline Model 3: patient variables ${ }^{2}$ & 0.36831 & 3.74755 & & & \\
\hline \multirow{10}{*}{$\begin{array}{l}\text { Full model } 4 \\
\text { (center and patient) }\end{array}$} & \multirow[t]{10}{*}{0.31850} & \multirow[t]{10}{*}{3.26345} & Large hospital & $0.40(0.21-0.79)$ & 0.008 \\
\hline & & & $\begin{array}{l}\text { Outpatient respiratory } \\
\text { nursing clinic available }\end{array}$ & $2.47(1.26-4.83)$ & 0.008 \\
\hline & & & Active smokers & $1.32(1.10-1.58)$ & 0.003 \\
\hline & & & Charlson index $\geq 3$ & $1.35(1.15-1.59)$ & $<0.001$ \\
\hline & & & $\begin{array}{l}\text { Number of hospital admissions } \\
\text { in the last year } \geq 1\end{array}$ & $6.33(5.02-7.98)$ & $<0.001$ \\
\hline & & & GesEPOC phenotype & & \\
\hline & & & Not exacerbator (reference) & & \\
\hline & & & Exacerbator & $0.79(0.61-1.101)$ & 0.063 \\
\hline & & & Missing & $0.36(0.29-0.44)$ & $<0.001$ \\
\hline & & & $\begin{array}{l}\text { To be taken care in specialized } \\
\text { COPD outpatient clinic) }\end{array}$ & $2.10(1.56-2.72)$ & $<0.001$ \\
\hline \multicolumn{6}{|c|}{$\begin{array}{l}\text { 1: included variables in the final center model: large hospital and outpatient respiratory nursing clinic available } \\
\text { 2: included variables in the final patient model: active smokers, Charlson index } \geq 3 \text {, number of hospital admissions } \\
\text { in the last year } \geq 1 \text {, to be taken care in specialized COPD outpatient clinic and GesEPOC phenotype exacerbator. }\end{array}$} \\
\hline \multicolumn{6}{|c|}{ Adherence to good clinical practice criteria in COPD evaluation ( $\geq 4$ criteria fulfilling) } \\
\hline Empty model 1 & 0.30343 & 3.13266 & & & \\
\hline Model 2: center variables ${ }^{1}$ & 0.26684 & 2.83994 & & & \\
\hline Model 3: patient variables ${ }^{2}$ & 0.29100 & 3.02953 & & & \\
\hline \multirow[t]{17}{*}{ Full model 4 (center and patient) } & 0.24413 & 2.67316 & Respiratory ward not available (reference) & & \\
\hline & & & Respiratory ward $<20$ beds & $7.09(2.53-9.90)$ & $<0.001$ \\
\hline & & & Respiratory ward $\geq 20$ beds & $3.00(1.37-6.60)$ & 0.006 \\
\hline & & & Age $\leq 55$ & $1.58(1.19-2.09)$ & 0.001 \\
\hline & & & Sex (male) & $0.77(0.61-0.96)$ & 0.022 \\
\hline & & & Charlson index $\geq 3$ & $0.80(0.68-0.94)$ & 0.008 \\
\hline & & & FEV1 $<50 \%$ & $1.68(1.42-1.99)$ & $<0.001$ \\
\hline & & & Dyspnea (MRC-m) & & \\
\hline & & & 0-1 (reference) & & \\
\hline & & & $\geq 2$ & $1.39(1.13-1.72)$ & 0.002 \\
\hline & & & Missing & $0.69(0.51-0.93)$ & 0.017 \\
\hline & & & Level of dyspnea not referred to & $0.65(0.49-0.86)$ & 0.003 \\
\hline & & & GesEPOC phenotype & & \\
\hline & & & Non-exacerbator (reference) & & \\
\hline & & & Exacerbator & $1.16(0.93-1.44)$ & 0.185 \\
\hline & & & Missing & $0.17(0.14-0.21)$ & $<0.001$ \\
\hline & & & Treatment at a specialized COPD outpatient clinic & $3.25(2.49-4.23)$ & $<0.001$ \\
\hline
\end{tabular}


Table 4 Multilevel logistic regression models of the variability in adherence to good clinical practice criteria for three categories: clinical evaluation of the patient, disease evaluation and therapeutic interventions (Continued)

\begin{tabular}{|c|c|c|c|c|c|}
\hline & $\begin{array}{l}\text { Intra-class } \\
\text { correlation (ICC) }\end{array}$ & $\begin{array}{l}\text { Median Odds } \\
\text { Ratio (MOR) }\end{array}$ & Variables & $\begin{array}{l}\text { Adjusted OR } \\
(95 \% \mathrm{Cl})\end{array}$ & $p$ \\
\hline \multicolumn{6}{|c|}{$\begin{array}{l}\text { 1: variables included in the final center model: in-patient respiratory clinic } \geq 20 \text { present and specialized COPD outpatient clinic available. } \\
\text { 2: variables included in the final patient model: age } \leq 55 \text {, gender (male), Charlson index } \geq 3 \text {, FEV } 1<50 \% \text {, dyspnea, } \\
\text { GesEPOC exacerbator phenotype and being treated in specialized COPD outpatient clinic. }\end{array}$} \\
\hline \multicolumn{6}{|c|}{ Adherence to good clinical practice criteria in therapeutic intervention ( $\geq 3$ criteria fulfilled) } \\
\hline Empty model 1 & 0.52169 & 6.09155 & & & \\
\hline Model 2: center variables ${ }^{1}$ & 0.46935 & 5.08927 & & & \\
\hline Model 3: patient variables ${ }^{2}$ & 0.49994 & 5.64024 & & & \\
\hline \multirow[t]{11}{*}{ Full model 4 (center and patient) } & 0.44731 & 4.74211 & University hospital & $0.26(0.08-0.85)$ & 0.026 \\
\hline & & & Outpatient respiratory nursing clinic availability & $3.69(1.50-9.11)$ & 0.005 \\
\hline & & & Age $\leq 55$ & $0.60(0.42-0.86)$ & $<0.005$ \\
\hline & & & Sex (male) & $0.72(0.55-0.93)$ & 0.014 \\
\hline & & & Charlson index $\geq 3$ & $1.19(0.99-1.42)$ & 0.062 \\
\hline & & & Number of hospital admissions in the last year $\geq 1$ & $1.71(1.38-2.11)$ & $<0.001$ \\
\hline & & & GesEPOC phenotype & & \\
\hline & & & Non-exacerbator (reference) & & \\
\hline & & & Exacerbator & $0.90(0.71-1.15)$ & 0.404 \\
\hline & & & Missing & $0.36(0.29-0.46)$ & $<0.001$ \\
\hline & & & Treatment at a specialized COPD outpatient clinic & $2.61(2.01-3.40)$ & $<0.001$ \\
\hline \multicolumn{6}{|c|}{$\begin{array}{l}\text { 1: variables included in the final center model: university hospital and outpatient respiratory nursing clinic availability } \\
\text { 2: variables included in the final patient model: age } \leq 55 \text {, gender (male), Charlson index } \geq 3 \text {, number of hospital admissions } \\
\text { in the last year } \geq 1 \text {, GesEPOC exacerbator phenotype and being treated in specialized COPD outpatient clinic }\end{array}$} \\
\hline
\end{tabular}

hospital's resources are potential components of the unexplained variation in outcomes. A greater number of medical and nursing staff was identified as a protective factor for intra-hospital mortality. In AUDIPOC Spain [12, 20], the large hospital COPD volume and the number of COPD patients admitted to the hospital the year prior to admission was identified as a predictor of a favourable outcome.

In our study, a large component of center-related variance remained unexplained, suggesting that the clinical profile of patients included in the study also varied markedly among hospitals. It is important to remember that recommendation guidelines are evidence-based and aimed to systemize medical care, but the clinical presentation of COPD is variable [21].

Our study has several strengths and limitations. The main strength is its sample size that accounts for $39 \%$ of the Spanish population. Nevertheless, the limitations to be considered are the fact that the selection of participating centers was not random and hospital participation was voluntary based on their interest to participate. Also, clinical records were used as the data source, so some missing and inconsistent values were unavoidable. Despite these limitations, we believe that this dataset represents the largest available comparative survey of Spanish centers.

\section{Conclusions}

High variability was observed among hospitals in terms of medical care. Some of the patient's characteristics (airflow obstruction, degree of dyspnea, exacerbation risk, presence of comorbidities) and the type of respiratory clinic in which the patient was treated (specialized COPD outpatient clinic) were identified as factors associated with a better adherence to recommendations, though a great part of the variability among center cannot be explained. This suggests that there is a significant inconsistency among centers in the implementation of clinical guidelines.

This information must be accounted for by health care professionals and administrators, in order to establish better clinical practice by means of the medical care in the specialized COPD outpatient clinic and the implementation of evidence-based best clinical practice guidelines that could facilitate a uniform approach to COPD patients as outpatients, thereby both improving patient outcomes and optimizing medical resources. 


\section{Appendix 1}

Table 5 The inclusion criteria and exclusion criteria

\begin{tabular}{ll}
\hline The inclusion criteria & - patients aged $\geq 40$ years \\
& - smokers or ex-smokers (of at least 10 pack-years) \\
& - COPD diagnosed on the basis of spirometric tests (FEV1/FVC post-bronchodilation $<0.7$ or FEV1/FVC pre-bronchodilation \\
& $<0.7$ and FEV1 $\geq 80 \%$, if there is no bronchodilation reversibility testing available \\
The exclusion criteria & - lack of follow-up for at least 1 year in a respiratory outpatient clinic \\
& - participating in a clinical trial
\end{tabular}

\section{Appendix 2}

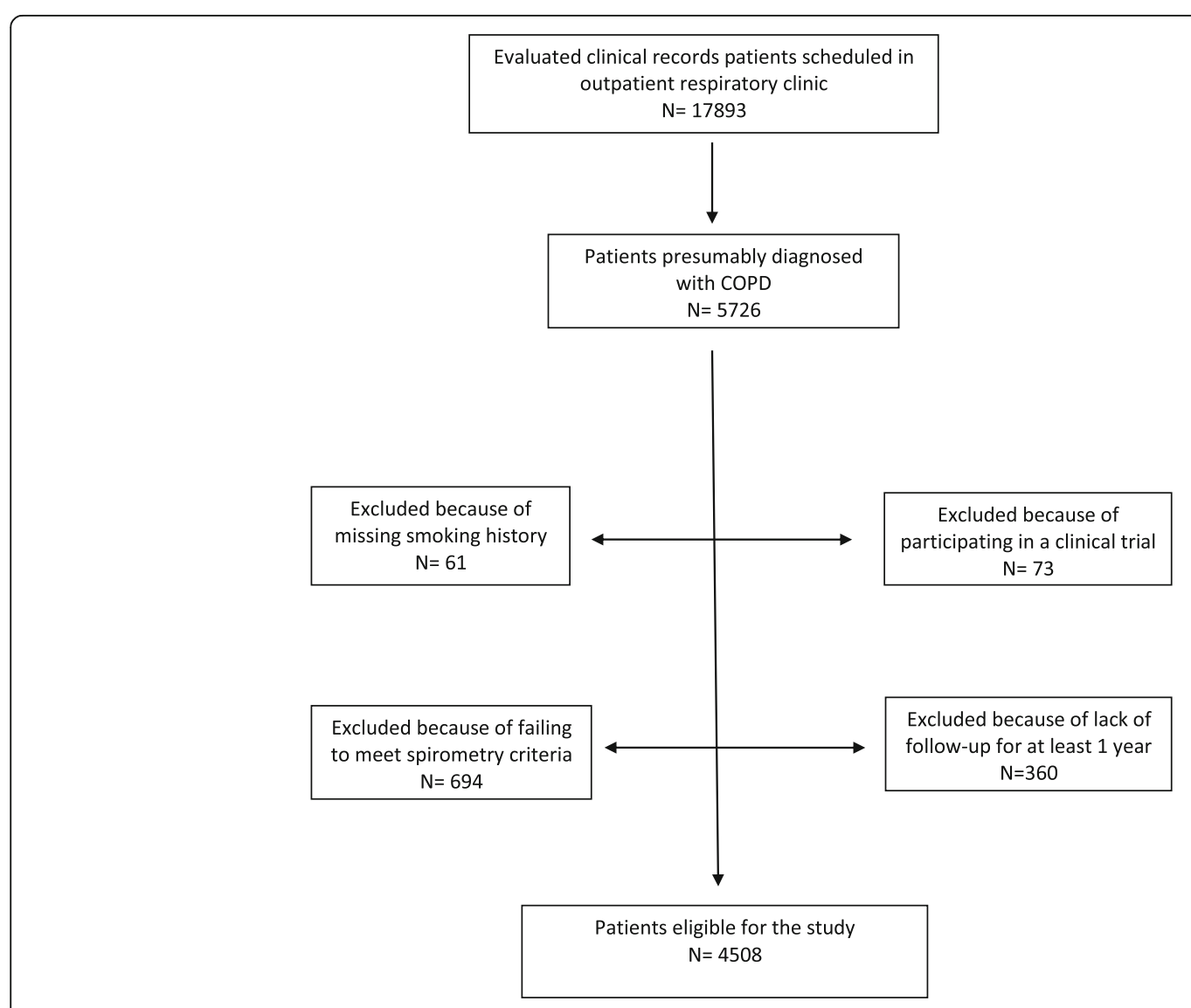

Fig. 1 The sampling process is described in a STROBE flow chart 


\section{Appendix 3}

Table 6 Participating hospitals and catchment population by Autonomous Community

\begin{tabular}{|c|c|c|c|c|}
\hline Region of Spain & $\begin{array}{l}\text { Number of participating } \\
\text { hospitals }\end{array}$ & $\begin{array}{l}\text { Population assigned for } \\
\text { admission }\end{array}$ & $\begin{array}{l}\text { Population of the } \\
\text { region }\end{array}$ & $\begin{array}{l}\text { Catchment population of the EPOCONSUL } \\
\text { study (\%) }\end{array}$ \\
\hline Andalucía & 10 & 2.784 .083 & 8.424 .102 & 33 \\
\hline Aragón & 2 & 597.000 & 1.346 .293 & 44.3 \\
\hline Asturias & 1 & 250.000 & 1.081 .487 & 23.1 \\
\hline Islas Baleares & 2 & 575.000 & 1.113 .114 & 51.6 \\
\hline País Vasco & 4 & 1.285 .000 & 2.184 .606 & 58.8 \\
\hline Islas Canarias & 1 & 700.000 & 2.126 .769 & 32.9 \\
\hline Cantabria & 2 & 395.000 & 593.121 & 66.6 \\
\hline $\begin{array}{l}\text { Castilla y la } \\
\text { Mancha }\end{array}$ & 4 & 1.186 .014 & 2.115 .334 & 56 \\
\hline Castilla y León & 4 & 1.119 .086 & 2.558 .463 & 43.7 \\
\hline Cataluña & 5 & 1.657 .000 & 7.539 .618 & 22 \\
\hline Extremadura & 1 & 273.977 & 1.109 .367 & 24.7 \\
\hline Galicia & 2 & 970.000 & 2.795 .422 & 34.7 \\
\hline Madrid & 11 & 3.484 .995 & 6.489 .680 & 53.7 \\
\hline Murcia & 3 & 770.175 & 1.470 .069 & 52.3 \\
\hline Navarra & 1 & 517.020. & 642.051 & 80.5 \\
\hline Valencia & 6 & 1.540 .000 & 5.117 .190 & 30 \\
\hline TOTAL & 59 & 18.104 .350 & 46.064 .635 & 39.3 \\
\hline
\end{tabular}

Data are presented as Numbers. The percentages refer to the total population number

There was no participating hospital in La Rioja, the $17^{\text {th }}$ Autonomous Community in Spain

\section{Appendix 4}

\section{Participants investigators in EPOCONSUL study}

Andalucía: Jose Luis Rojas Box, H. de Alta Resolución de Écija, Sevilla. Jose Domingo Garcia Jimenez, H. de Alta Resolución de Utrera, Sevilla. Adolfo Domenech del Rio, Ana Muñoz. H. Carlos Hayas, Málaga. Antonia Soto Venegas, H. San Juan de la Cruz, Úbeda, Jaén. Aurelio Arnedillo Muñoz. H. U. Puerta del Mar, Cádiz. Agustín Valido Morales. H. Virgen de Macarena. Sevilla. Jose Velasco Garrido, Carlos Rueda Ríos, Macarena Arroyo Varela H. Virgen de la Victoria. Málaga. Francisco Ortega Ruiz, Eduardo Marquez Martin, Carmen Calero Acuña, H. Virgen del Rocio, Sevilla. Francisco Luis Garcia Gil, H. U Reina Sofia, Córdoba.

Aragón: Joaquin Carlos Costan Galicia, H. Clínico U. Lozano Blesa, Zaragoza. Ana Boldova Loscertales, $\mathrm{H}$. Royo Villanova, Zaragoza.

Asturias: Cristina Martinez González, Rosirys Guzman Taveras, H. U. Central de Asturias, Oviedo.

Murcia: Juan Luis De la Torre Alvaro, H. U Santa Lucia, Cartagena, $M^{a}$ Jesus Avilés Ingles, H. General U. Reina Sofia, Murcia. Rubén Andújar Espinosa, H.U. Virgen de la Arrixaca, Murcia.

Canarias: Juan Manuel Palmero Tejera, Juan Marco Figueira Conçalves, H.U. Nuestra Señora de la Candelaria, Santa Cruz de Tenerife.
Cantabria: Ramon Agüero Balbín, Carlos Amado Diago, Beatriz Abascal Bolado.

H. Marqués de Valdecilla, Santander. Juan Luis Garcia Rivero, Marcelle Cohen Escovar, H. de Laredo, Santander.

Castilla y la Mancha: Francisco Javier Callejas González. Complejo hospitalario universitario de Albacete, Albacete. Angel Ortega Gonzalez. H Nuestra Señora del Prado, Talavera de la Reina, Toledo. Rosario Vargas Gonzalez, H. Virgen de la Luz, Cuenca. Encarnación López Gabaldón, Raul Hidalgo Carvajal, H. Virgen de la Salud, Toledo.

Castilla y León: Elena Bollo de Miguel, Silvia Fernández Huerga, Complejo Hospitalario Universitario de León. Ana Pueyo Bastida, Complejo Asistencial de Burgos, Burgos. Jesus R Hernández Hernández, Ruth Garcia García, H. Nuestra Señora de Sonsoles, Ávila. Miguel Barrueco Ferrero, Marco López Zuibizarreta, E. Consuelo Fernández, H. Universitario de Salamanca.

Cataluña: David De la Rosa Carrillo, H. Plató, Barcelona. Jordi Esplugas Abós, Noelia Pablos Mateos, H. Sant Joan de Déu, Martorell. Elena De Miguel Campos, H. Sant Joan de Despi, Barcelona. Pablo Rubinstein, Hospital General de Cataluña, Barcelona. Hernán Abraham Manrique Chávez, H Sagrat Cor, Barcelona. Miriam Barrecheguren, H. U. Vall d'Hebron, Barcelona. 
Valencia: Carmen Aguar Benito, H. de Arnau de Villanova, Valencia. Pablo Catalán Serra, H. de Requena, Requena. Eusebi Chiner Vives. H. U. de San Juan, Alicante. Juan Antonio Royo Prats. H. General de Castellón, Castellón de la Plana. Cristina Sabater Abad, Esther Verdejo Mengual, H. General Universitario de Valencia. Eva Martínez- Moragon, H. Universitario Dr. Peset, Valencia.

Extremadura: Francisca Lourdes Marquez Perez, H. U Santa Cristina, Badajoz.

Galicia: Alberto Fernandez Villar, Cristina Represas Represas, Ana Priegue Carrera, Complejo hospitalario de Vigo. Marina Blanco Aparicio, Pedro Jorge Marcos Rodriguez, H. U. Juan Canalejo, La Coruña.

Baleares: Federico Gonzalo Fiorentino, $\mathrm{M}^{\mathrm{a}}$ Magdalena Pan Naranjo, H. Son Espases, Palma de Mallorca. Antonia Fuster Gomila, H. Sant Llatzer, Palma de Mallorca.

Madrid: German Peces Barba, Felipe Villar Alvarez, Fundación Jimenez Diaz, Madrid. Carlos Jose Álvarez Martinez, H. 12 de Octubre, Madrid. Juan Luis
Rodriguez Hermosa, J.L. Álvarez Sala-Walther, Juan Rigüal Bobillo, Gianna Vargas Centanaro, H. Clinico San Carlos, Madrid. José Andrés García Romero de Tejada, H. U. Infanta Sofía, San Sebastián de los Reyes, Madrid. Javier Jareño, Sergio Campos Tellez. H. Central de la Defensa, Madrid. Raul Galera Martinez, H. La Paz. Rosa Mar Gómez Punter, Emma Vázquez Espinosa, H. La Princesa, Madrid. Esther Alonso Peces, H. Principe de Asturias, Alcalá de Henares, Madrid. Juan Manuel Diez Piña, Raquel Pérez Rojo, H. U. de Móstoles, Madrid. Luis Puente Maestu, Julia Garcia de Pedro. H. U. Gregorio Marañón, Madrid. Soledad Alonso Viteri, H. U de Torrejón, Torrejón de Ardoz, Madrid.

Navarra: Maria Hernandez Bonaga, Complejo Hospitalario de Navarra, Pamplona.

País Vasco: Maria Milagros Iriberri Pascual, $\mathrm{H}$ de Cruces, Baracaldo. Myriam Aburto Barrenechea, $\mathrm{H}$ de Galdakano. Sophe Garcia Fuika, Hospital Santiago Apostol, Vitoria. Patricia Sobradillo Ecenarro, Hospital Txagorritx, Basurto.

\section{Appendix 5}

Table 7 Logistic regression bivariate analysis (adherence to good clinical practice criteria in three categories: clinical evaluation of the patient, COPD evaluation and therapeutic interventions)

\begin{tabular}{|c|c|c|c|c|c|}
\hline Patients & $\begin{array}{l}\text { Clinical evaluation } \\
\geq 3 \text { criteria fulfilled } \\
\text { OR }(95 \% \mathrm{Cl})\end{array}$ & $p$ & Centers & $\begin{array}{l}\text { Clinical evaluation } \\
\geq 3 \text { criteria fulfilled } \\
\text { OR }(95 \% \text { Cl) }\end{array}$ & $p$ \\
\hline Age ( $\leq 55$ years) & $1.01(0.78-1.31)$ & 0.89 & Large hospital & $0.44(0.21-0.89)$ & 0.024 \\
\hline Sex (male) & $0.96(0.78-1.17)$ & 0.70 & University hospital & $0.32(0.12-0.82)$ & 0.018 \\
\hline Active smokers & $1.25(1.05-1.48)$ & 0.011 & Beds per center $\geq 500$ & $0.57(0.27-1.22)$ & 0.152 \\
\hline Dyspnea (MRC-m) & & & Respiratory ward not available & Reference & \\
\hline $0-1$ & & & Respiratory ward $<20$ beds & $0.90(0.24-3.32)$ & 0.876 \\
\hline$\geq 2$ & Reference & & Respiratory ward $\geq 20$ beds & $0.51(0.19-1.35)$ & 0.178 \\
\hline Missing & $1.02(0.83-1.26)$ & 0.797 & & & \\
\hline \multirow{2}{*}{$\begin{array}{l}\text { Level of dyspnea } \\
\text { not referred to }\end{array}$} & $0.24(0.19-0.31)$ & $<0.001$ & & & \\
\hline & $0.11(0.09-0.15)$ & $<0.001$ & & & \\
\hline FEV $1<50 \%$ & $1.24(1.07-1.43)$ & 0.004 & $\begin{array}{l}\text { Number of pulmonology } \\
\text { staff members } \geq 5\end{array}$ & $0.80(0.35-1.86)$ & 0.620 \\
\hline Charlson index $\geq 3$ & $1.45(1.25-1.69)$ & $<0.001$ & Pulmonology residents present & $0.90(0.41-1.99)$ & 0.806 \\
\hline $\begin{array}{l}\text { Number of hospital } \\
\text { admissions in the } \\
\text { last year } \geq 1\end{array}$ & $6.45(5.16-8.07)$ & $<0.001$ & $\begin{array}{l}\text { Number of annual outpatient respiratory visits } \\
\geq 10,000\end{array}$ & $0.45(0.17-1.19)$ & 0.109 \\
\hline GesEPOC phenotype & & & $\begin{array}{l}\geq 15 \text { min of follow-up at general } \\
\text { outpatient respiratory visit }\end{array}$ & $2.34(1.13-4.83)$ & 0.021 \\
\hline Non-exacerbator & Reference & & & & \\
\hline Exacerbator & $1.26(1.00-1.59)$ & 0.048 & & & \\
\hline Missing & $0.40(0.33-0.49)$ & $<0.001$ & & & \\
\hline Triple inhalation therapy & $1.01(0.87-1.17)$ & 0.872 & $\begin{array}{l}\text { Specialized COPD outpatient } \\
\text { clinic available }\end{array}$ & $1.07(0.51-2.22)$ & 0.850 \\
\hline \multirow[t]{2}{*}{$\begin{array}{l}\text { Treatment at a specialized COPD } \\
\text { outpatient clinic }\end{array}$} & $2.49(1.91-3.23)$ & $<0.001$ & $\begin{array}{l}\text { Outpatient respiratory nursing } \\
\text { clinic availability }\end{array}$ & $2.47(1.23-4.97)$ & 0.011 \\
\hline & & & $\begin{array}{l}\text { Inhalation technique } \\
\text { educational program } \\
\text { available }\end{array}$ & $1.61(0.73-3.55)$ & 0.234 \\
\hline
\end{tabular}


Table 7 Logistic regression bivariate analysis (adherence to good clinical practice criteria in three categories: clinical evaluation of the patient, COPD evaluation and therapeutic interventions) (Continued)

\begin{tabular}{|c|c|c|c|c|c|}
\hline Patients & $\begin{array}{l}\text { Clinical evaluation } \\
\geq 3 \text { criteria fulfilled } \\
\text { OR }(95 \% \text { Cl) }\end{array}$ & $p$ & Centers & $\begin{array}{l}\text { Clinical evaluation } \\
\geq 3 \text { criteria fulfilled } \\
\text { OR }(95 \% \text { Cl) }\end{array}$ & $p$ \\
\hline Patients & $\begin{array}{l}\text { COPD evaluation } \\
\geq 4 \text { criteria fulfilled } \\
\text { OR }(95 \% \text { Cl) }\end{array}$ & $p$ & Centers & $\begin{array}{l}\text { COPD evaluation } \\
\geq 4 \text { criteria fulfilled } \\
\text { OR ( } 95 \% \text { Cl) }\end{array}$ & $p$ \\
\hline Age ( $\leq 55$ years) & $1.68(1.31-2.14)$ & $<0.001$ & Large hospital & $1.25(0.66-2.39)$ & 0.484 \\
\hline Sex (male) & $0.75(0.61-0.92)$ & 0.006 & University hospital & $1.16(0.49-2.74)$ & 0.729 \\
\hline Active smokers & $1.01(0.82-0.85)$ & 0.824 & Beds per center $\geq 500$ & $1.65(0.85-3.20)$ & 0.136 \\
\hline Dyspnea (MRC-m) & & & Respiratory ward not available & Reference & \\
\hline $0-1$ & & & Respiratory ward $<20$ beds & $4.23(1.39-12.86)$ & 0.011 \\
\hline$\geq 2$ & Reference & & Respiratory ward $\geq 20$ beds & $2.23(0.96-5.19)$ & 0.062 \\
\hline Missing & $1.53(1.28-1.84)$ & $<0.001$ & & & \\
\hline \multirow{2}{*}{$\begin{array}{l}\text { Level of dyspnea } \\
\text { not referred to }\end{array}$} & $0.51(0.38-0.68)$ & $<0.001$ & & & \\
\hline & $0.54(0.41-0.69)$ & $<0.001$ & & & \\
\hline FEV $1<50 \%$ & $1.80(1.55-2.08)$ & $<0.001$ & $\begin{array}{l}\text { Number of pulmonology } \\
\text { staff members } \geq 5\end{array}$ & $1.44(0.69-3.00)$ & 0.324 \\
\hline Charlson index $\geq 3$ & $0.80(0.69-0.92)$ & 0.003 & Pulmonology residents present & $1.69(0.85-3.37)$ & 0.129 \\
\hline $\begin{array}{l}\text { Number of hospital } \\
\text { admissions in the } \\
\text { last year } \geq 1\end{array}$ & $1.30(1.10-1.55)$ & 0.002 & $\begin{array}{l}\text { Number of annual outpatient } \\
\text { respiratory visits } \geq 10,000\end{array}$ & $1.82(0.78-4.25)$ & 0.165 \\
\hline \multicolumn{3}{|l|}{ GesEPOC phenotype } & \multirow{4}{*}{$\begin{array}{l}\geq 15 \text { min of follow-up at } \\
\text { general outpatient } \\
\text { respiratory visit }\end{array}$} & \multirow[t]{4}{*}{$1.06(0.54-2.09)$} & \multirow[t]{4}{*}{0.845} \\
\hline Non-exacerbator & Reference & & & & \\
\hline Exacerbator & $1.38(1.13-1.69)$ & 0.001 & & & \\
\hline Missing & $0.16(0.13-0.20)$ & $<0.001$ & & & \\
\hline Triple inhalation therapy & $1.37(1.18-1.60)$ & $<0.001$ & $\begin{array}{l}\text { Specialized COPD outpatient } \\
\text { clinic available }\end{array}$ & $1.77(0.94-3.31)$ & 0.073 \\
\hline \multirow[t]{2}{*}{$\begin{array}{l}\text { Treatment at a specialized COPD } \\
\text { outpatient clinic }\end{array}$} & \multirow[t]{2}{*}{$4.44(3.48-5.68)$} & \multirow[t]{2}{*}{$<0.001$} & $\begin{array}{l}\text { Outpatient respiratory nursing } \\
\text { clinic availability }\end{array}$ & $1.57(0.83-2.95)$ & 0.158 \\
\hline & & & $\begin{array}{l}\text { Inhalation technique } \\
\text { educational program } \\
\text { available }\end{array}$ & $1.91(0.97-3.75)$ & 0.058 \\
\hline Patients & $\begin{array}{l}\text { Therapeutic intervention } \\
\geq 3 \text { criteria fulfilled } \\
\text { OR }(95 \% \text { Cl) }\end{array}$ & p & Centers & $\begin{array}{l}\text { Therapeutic intervention } \\
\geq 3 \text { criteria fulfilled } \\
\text { OR }(95 \% \text { Cl) }\end{array}$ & $p$ \\
\hline Age ( $\leq 55$ years) & $0.62(0.44-0.87)$ & 0.007 & Large hospital & $0.46(0.16-1.25)$ & 0.130 \\
\hline Sex (male) & $0.79(0.61-1.02)$ & 0.074 & University hospital & $0.32(0.08-1.17)$ & 0.087 \\
\hline Active smokers & $0.98(0.80-1.20)$ & 0.894 & Beds per centre $\geq 500$ & $0.78(0.27-2.25)$ & 0.656 \\
\hline Dyspnea (MRC-m) & & & Respiratory ward not available & Reference & \\
\hline $0-1$ & Reference & & & $0.26(0.04-1.66)$ & 0.158 \\
\hline$\geq 2$ & $1.10(0.90-1.35)$ & 0.317 & Respiratory ward $<20$ beds & $0.32(0.08-1.19)$ & 0.090 \\
\hline Missing & $0.09(0.05-0.15)$ & $<0.001$ & Respiratory ward $\geq 20$ beds & & \\
\hline $\begin{array}{l}\text { Level of dyspnea } \\
\text { not referred to }\end{array}$ & $0.24(0.17-0.34)$ & $<0.001$ & & & \\
\hline FEV $1<50 \%$ & $1.32(1.07-1.63)$ & 0.008 & $\begin{array}{l}\text { Number of pulmonology } \\
\text { staff members } \geq 5\end{array}$ & $0.41(0.13-1.27)$ & 0.125 \\
\hline Charlson index $\geq 3$ & $1.23(1.04-1.46)$ & 0.016 & Pulmonology residents present & $0.87(0.29-2.61)$ & 0.813 \\
\hline $\begin{array}{l}\text { Number of hospital } \\
\text { admissions in the }\end{array}$ & $1.83(1.50-2.23)$ & 0.000 & $\begin{array}{l}\text { Number of annual outpatient } \\
\text { respiratory visits } \geq 10,000\end{array}$ & $0.29(0.08-1.06)$ & 0.062 \\
\hline
\end{tabular}


Table 7 Logistic regression bivariate analysis (adherence to good clinical practice criteria in three categories: clinical evaluation of the patient, COPD evaluation and therapeutic interventions) (Continued)

\begin{tabular}{|c|c|c|c|c|c|}
\hline Patients & $\begin{array}{l}\text { Clinical evaluation } \\
\geq 3 \text { criteria fulfilled } \\
\text { OR }(95 \% \text { Cl) }\end{array}$ & $p$ & Centers & $\begin{array}{l}\text { Clinical evaluation } \\
\geq 3 \text { criteria fulfilled } \\
\text { OR }(95 \% \mathrm{Cl})\end{array}$ & $p$ \\
\hline GesEPOC phenotype & & & \multirow{4}{*}{$\begin{array}{l}\geq 15 \text { min of follow-up at } \\
\text { general outpatient } \\
\text { respiratory visit }\end{array}$} & \multirow[t]{4}{*}{$1.09(0.37-3.20)$} & \multirow[t]{4}{*}{0.862} \\
\hline Non-exacerbator & Reference & & & & \\
\hline Exacerbator & $1.10(0.87-1.38)$ & 0.414 & & & \\
\hline Missing & $0.37(0.30-0.46)$ & $<0.001$ & & & \\
\hline Triple inhalation therapy & $1.02(0.85-1.21)$ & 0.812 & Specialized COPD outpatient clinic available & $2.14(0.79-5.79)$ & 0.133 \\
\hline \multirow[t]{2}{*}{$\begin{array}{l}\text { Treatment at a specialized } \\
\text { COPD outpatient clinic }\end{array}$} & $2.95(2.28-3.83)$ & $<0.001$ & $\begin{array}{l}\text { Outpatient respiratory nursing clinic } \\
\text { availability }\end{array}$ & $3.36(1.27-8.84)$ & 0.014 \\
\hline & & & $\begin{array}{l}\text { Inhalation technique educational } \\
\text { program available }\end{array}$ & $3.55(1.25-10.07)$ & 0.017 \\
\hline
\end{tabular}

\section{Abbreviations}

BMl: Body mass index; COPD: Chronic obstructive pulmonary disease; CPG: Clinical practice guidelines; CSI: Inhaled corticosteroids; GesEPOC: Spanish National Guideline for COPD care; ICC: Intra-cluster correlation coefficient; IHR: Inter-hospital range; IQR: Interquartile range; LABA: Long-acting beta-2 agonists; LAMA: Long-acting antimuscarinic agents; MOR: Median odds ratio; OR: Crude odds ratio

\section{Acknowledgements}

No aplicable (Anyone who contributed towards the article who does not meet the criteria for authorship).

\section{Funding}

This study has been promoted and sponsored by the SEPAR. We thank Boehringer Ingelheim for its financial support to carry out the study. The financing entities did not participate in the design of the study, data collection, analysis, publication or preparation of this manuscript.

\section{Availability of data and materials}

The datasets used and/or analysed during the current study are available from the corresponding author on reasonable request.

\section{Declarations}

The protocol was approved by the Ethics Committee of the Hospital Clínico San Carlos (Madrid, Spain; internal code 14/030-E). According to current research laws in Spain, the ethics committee at each participating hospital evaluated and agreed to the study protocol. The need for informed consent was waived due to the non-interventional nature of the study, the anonymization of data and the need to blindly evaluate the clinical performance. This circumstance was clearly explained in the protocol, and the ethical committees approved this procedure.

\section{Authors' contributions}

MCR, JLLC, BAN, JBS, JJSC, JMRG form the study's Scientific Committee. MEFF carried out the statistical analysis. JRH contributed substantially to data analysis and results interpretation. MCR designed the study and wrote the manuscript. The rest of the authors recruited patients and reviewed the manuscript. All authors contributed to data analysis, drafting and revising the paper, and agree to be accountable for all aspects of the work.

\section{Consent for publication}

No aplicable (does not contain any individual persons data and does not report on or involve the use of any animal or human data or tissue).

\section{Competing interests}

The authors declare that they have no competing interests.

\section{Publisher's Note}

Springer Nature remains neutral with regard to jurisdictional claims in published maps and institutional affiliations.

\section{Author details}

${ }^{1}$ Pulmonary Department, Research Institute of Hospital Clínico San Carlos (IdISSC), Faculty of Medicine, University Complutense of Madrid, C/ Martin Lagos s/n, 28040 Madrid, Spain. ²Unidad Médico-Quirúrgica de Enfermedades Respiratorias, Instituto de Biomedicina de Sevilla (IBiS), Hospital Universitario Virgen del Rocío/Universidad of Sevilla, Sevilla, Spain. ${ }^{3}$ CIBER de Enfermedades Respiratorias (CIBERES), Instituto de Salud Carlos III, Madrid, Spain. ${ }^{4}$ Pulmonary Department, Hospital of Arnau de Villanova, Valencia, Spain. ${ }^{5}$ Pulmonary Department, Hospital of Alta Resolución de Loja, Granada, Spain. ${ }^{6}$ Research Institute of Hospital University La Princesa (IISP), University Autónoma of Madrid, Madrid, Spain. ${ }^{7}$ Pulmonary Department, $\mathrm{H}$. Universitario Príncipe de Asturias, Alcalá de Henares, Madrid, Spain. ${ }^{8}$ UGC de Medicina Preventiva, Research Institute of Hospital Clínico San Carlos (IdISSC), Hospital Clínico San Carlos, Faculty of Medicine, University Complutense of Madrid, Madrid, Spain.

Received: 9 October 2017 Accepted: 19 November 2017 Published online: 02 December 2017

\section{References}

1. Fromer L. Implementing chronic care for COPD: planned visits, care coordination, and patient empowerment for improved outcomes. Int J Chron Obstruct Pulmon Dis. 2011;6:605-14.

2. Chapman KR, Mannino DM, Soriano JB, Vermeire PA, Buist AS, Thun MJ, et al. Epidemiology and costs of chronic obstructive pulmonary disease. Eur Respir J. 2006:27:188-207.

3. Perera PN, Armstrong EP, Sherrill DL, Skrepnek GH. Acute exacerbations of COPD in the United States: inpatient burden and predictors of costs and mortality, COPD. 2012;9:131-41

4. Miravitlles M, Soler-Cataluña JJ, Calle M, Molina J, Almagro P, Quintano JA, et al. Spanish guideline for COPD (GesEPOC). Update 2014. Arch Bronconeumol. 2014; 50(Suppl 1):1-16.

5. Vestbo J, Hurd SS, Agusti AG, Jones PW, Vogelmeier C, Anzueto A, et al. Global strategy for the diagnosis, management, and prevention of chronic obstructive pulmonary disease: GOLD executive summary. Am J Respir Crit Care Med. 2013; 187:347-65

6. Celli BR, MacNee W, ATS/ERS Task Force. Standards for the diagnosis and treatment of patients with COPD: a summary of the ATS/ERS position paper. Eur Respir J. 2004:23:932-46.

7. Soler-Cataluña JJ, Calle M, Cosío BG, Marín JM, Monsó E, Alfageme I, SEPAR Quality Assistance Committee, SEPAR EPOC Work Area. Health-care quality standards in chronic obstructive pulmonary disease. Arch Bronconeumol. 2009; 45:196-203.

8. Miravitlles M, Vogelmeier C, Roche N, Halpin D, Cardoso J, Chuchalin AG, et al. A review of national guidelines for management of COPD in Europe. Eur Respir J. 2016;47:625-37.

9. Wong GW, Miravitlles M, Chisholm A, Krishnan JA. Respiratory guidelines which real world? Ann Am Thorac Soc. 2014;11(Suppl 2):S85-91.

10. Flottorp SA. Using audit and feedback to health professionals to improve the quality and safety of health care. Copenhagen European Observatory on Health Systems and Policies: WHO. regional office for Europe; 2010. IX, p. 42. 
11. Roberts CM, Lopez-Campos JL, Pozo-Rodriguez F, Hartl S, European COPD Audit team. European hospital adherence to GOLD recommendations for chronic obstructive pulmonary disease (COPD) exacerbation admissions. Thorax. 2013;68:1169-71.

12. Pozo-Rodríguez F, López-Campos JL, Álvarez-Martínez CJ, Castro-Acosta A, Agüero R, Hueto J, AUDIPOC Study Group, et al. Clinical audit of COPD patients requiring hospital admissions in Spain: AUDIPOC study. PLoS One. 2012;7:e42156.

13. Lopez-Campos JL, Hartl S, Pozo-Rodriguez F, Roberts CM, European COPD Audit team. Variability of hospital resources for acute care of COPD patients: the European COPD audit. Eur Respir J. 2014;43:754-62.

14. Nardini S, Cicchitto G, De Benedetto F, Donner CF, Polverino M, Sanguinetti CM, et al. Audit on the appropriateness of integrated COPD management: the "ALT-BPCO" project. Multidiscip Respir Med. 2014;9:40.

15. Jones R, Roberts M. National cohort data from Sweden to the national COPD audit in England and Wales: grand designs for quality improvement. Prim Care Respir J. 2014;23:7-8.

16. Ställberg B, Janson C, Johansson G, Larsson K, Stratelis G, Telg G, et al. Management, morbidity and mortality of COPD during an 11-year period: an observational retrospective epidemiological register study in Sweden (PATHOS). Prim Care Respir J. 2014;23:38-45.

17. Kinnula VL, Vasankari T, Kontula E, Sovijarvi A, Saynajakangas O, Pietinalho A. The 10-year COPD Programme in Finland: effects on quality of diagnosis, smoking, prevalence, hospital admissions and mortality. Prim Care Respir J. 2011;20:178-83.

18. López-Campos JL, Abad Arranz M, Calero-Acuña C, Romero-Valero F, Ayerbe-García R, Hidalgo-Molina A, et al. Guideline adherence in outpatient clinics for chronic obstructive pulmonary disease: results from a clinical audit. PLoS One. 2016;11:e0151896.

19. Calle M, Alcázar B, Soriano JB, Soler-Cataluña JJ, Rodríguez JM, Fuentes M, et al. Clinical audit of COPD in outpatient respiratory clinics in Spain: the EPOCONSUL study. Int J Chron Obstruct Pulm Dis. 2017;12:417-26.

20. Pozo-Rodríguez F, Castro-Acosta A, Alvarez CJ, López-Campos JL, Forte A, López-Quilez A, AUDIPOC Study Group, et al. Determinants of betweenhospital variations in outcomes for patients admitted with COPD exacerbations: findings from a nationwide clinical audit (AUDIPOC) in Spain. Int J Clin Pract. 2015:69:938-47.

21. Lugtenberg M, Burgers JS, Clancy C, Westert GP, Schneider EC. Current guidelines have limited applicability to patients with comorbid conditions: a systematic analysis of evidence-based guidelines. PLoS One. 2011;6:e25987.

\section{Submit your next manuscript to BioMed Central and we will help you at every step:}

- We accept pre-submission inquiries

- Our selector tool helps you to find the most relevant journal

- We provide round the clock customer support

- Convenient online submission

- Thorough peer review

- Inclusion in PubMed and all major indexing services

- Maximum visibility for your research

Submit your manuscript at www.biomedcentral.com/submit

) Biomed Central 